\title{
Network Meta-analysis of Progression-Free Survival and Overall Survival in First-Line Treatment of BRAF Mutation-Positive Metastatic Melanoma
}

\author{
Jordan Amdahl · Lei Chen · Thomas E. Delea
}

Received: May 18, 2016/Published online: September 27, 2016

(C) The Author(s) 2016. This article is published with open access at Springerlink.com

\begin{abstract}
Introduction: The present study aimed to inform an economic evaluation of dabrafenib and trametinib combination as first-line treatment of metastatic melanoma in a Canadian setting. A network meta-analysis was conducted to estimate hazard ratios (HRs) for progression-free survival (PFS)and overall survival (OS) of dabrafenib plus trametinib versus other first-line treatments of BRAF mutation-positive metastatic melanoma including dabrafenib, trametinib, vemurafenib, ipilimumab, and dacarbazine (DTIC).
\end{abstract}

Methods: HRs for PFS and OS were from randomized controlled trials identified from

Enhanced content To view enhanced content for this article go to http://www.medengine.com/Redeem/ 6595F0606BD7B8E7.

Electronic supplementary material The online version of this article (doi:10.1007/s40487-016-0030-2) contains supplementary material, which is available to authorized users.

J. Amdahl · T. E. Delea ( $\varangle)$

Policy Analysis Inc. (PAI), 4 Davis Court, Brookline, MA 02445, USA

e-mail: tdelea@pai2.com

L. Chen

Novartis Pharmaceuticals, East Hanover, NJ, USA systematic literature reviews. HRs for PFS and OS (adjusted for crossover as appropriate) were analyzed using multivariate and univariate Bayesian network meta-analysis.

Results: In multivariate network-meta analyses (HRs for PFS and OS estimated simultaneously to account for the correlation of treatment effects on PFS and OS), HRs (95\% credible interval) for PFS and OS favored dabrafenib plus trametinib [PFS: $0.23(0.18-0.29)$ versus DTIC, $0.32(0.24-0.42)$ versus ipilimumab plus DTIC, $0.52(0.32-0.83)$ versus trametinib, 0.57 (0.48-0.69) versus vemurafenib, and 0.59 (0.50-0.71) versus dabrafenib]; OS [0.41 (0.29-0.56) versus DTIC, $0.52 \quad(0.38-0.71)$ versus ipilimumab plus DTIC, 0.68 (0.47-0.95) versus trametinib, $0.69(0.57-0.84)$ versus vemurafenib, and $0.72(0.60-0.85)$ versus dabrafenib]. The beneficial effects on OS of dabrafenib plus trametinib versus ipilimumab plus DTIC and versus trametinib were attenuated when HRs were estimated using univariate network meta-analysis (HRs for PFS and OS estimated separately).

Conclusion: This analysis demonstrates improved PFS and OS with dabrafenib + trametinib versus dabrafenib, 
trametinib, vemurafenib, ipilimumab plus DTIC, and DTIC as first-line treatment for patients with BRAF mutation-positive metastatic melanoma.

Funding: Novartis Pharmaceuticals.

Keywords: Clinical trials; Melanoma; Network meta-analysis

\section{INTRODUCTION}

The treatment of metastatic melanoma has been transformed in recent years by the introduction of immune checkpoints inhibitors and therapies targeted on the MAP kinase (MAPK) pathway. Immune checkpoint inhibitors block proteins on cytotoxic T-cells or cancer cells which inhibit T cell signaling, such as the cytotoxic lymphocyte-associated protein 4 (CTLA-4) or the programmed death-1 (PD-1) protein, thus reactivating an immune system response against tumor cells. Ipilimumab, an anti-CTLA-4 antibody, was the first immune checkpoint inhibitor to be approved in melanoma. In controlled trials, ipilimumab has demonstrated consistent activity against melanoma in pretreated and chemotherapy-naïve patients [1-3]. More recently, two novel anti-PD-1 immune checkpoint inhibitors, pembrolizumab and nivolumab, have been approved based on randomized controlled trials demonstrating improved outcomes versus ipilimumab alone [4-6].

Dysregulation of BRAF signaling in the MAPK pathway is a key driver of metastatic melanoma [7]. Two inhibitors of the BRAF protein, vemurafenib and dabrafenib, have been approved for treatment of patients with BRAF V600 mutation-positive unresectable stage IIIC or stage IV (advanced or metastatic) melanoma.
These approvals were based on the results of the BRIM-3 (ClinicalTrials.gov identifier: NCT01006980) and BREAK-3 (ClinicalTrials.gov identifier: NCT01227889) randomized controlled trials, respectively, which demonstrated improved outcomes versus dacarbazine (DTIC) in front-line treatment of BRAF V600 mutation-positive unresectable or metastatic melanoma [8-14]. Trametinib is a highly potent and selective small-molecule inhibitor of the MEK kinase that is downstream of and activated by BRAF in the MAPK pathway. Trametinib has been shown to be more effective than chemotherapy in treatment-naïve or previously treated patients with BRAF V600 mutation-positive unresectable or metastatic melanoma [15]. These results, as well as improved understanding of the mechanisms of resistance to targeted therapies, have led to the exploration of combination approaches to targeted therapy. The efficacy and safety of dabrafenib in combination with trametinib has been evaluated in two phase 3 trials, the COMBI-d (ClinicalTrials.gov identifier: NCT01584648) and COMBI-v (ClinicalTrials.gov identifier: NCT01597908) trials, as well as the phase 2 BRF113220 (ClinicalTrials.gov identifier: NCT01072175) trial [16-21]. Results of these trials have demonstrated that dabrafenib plus trametinib improves progression-free survival (PFS) and overall survival (OS) compared with dabrafenib monotherapy or vemurafenib monotherapy treatment-naïve patients with BRAF V600 mutation-positive unresectable or metastatic melanoma.

Economic evaluations of novel treatments such as dabrafenib plus trametinib require estimates of the relative treatment effects versus other available therapies on PFS and OS. The objective of this study was to conduct a network meta-analysis of HRs for PFS and OS of 
dabrafenib plus trametinib versus other first-line treatments of BRAF mutation-positive advanced or metastatic melanoma for use in an economic evaluation of dabrafenib plus trametinib in this indication for submission to Canadian reimbursement authorities.

\section{METHODS}

This study was a network meta-analysis of HRs for PFS and OS reported in randomized controlled trials of first-line treatments for patients with BRAF mutation-positive advanced or metastatic melanoma. Comparators were limited to first-line treatments for patients with metastatic melanoma that were approved by Health Canada as of February 2015 and included dabrafenib plus trametinib, dabrafenib, vemurafenib, trametinib, ipilimumab, and DTIC. Nivolumab, pembrolizumab, and cobimetinib plus vemurafenib were not included in the analysis as these treatments were not yet approved of that date.

Trials included in the analysis were identified from two systematic literature reviews. Details regarding the reviews have been published previously [22, 23]. Both reviews followed the Preferred Reporting Items for Systematic Reviews and Meta-analysis (PRISMA) guidelines [24]. The first review was undertaken to evaluate efficacy, safety, and tolerability of dabrafenib and trametinib monotherapy versus other first-line treatments for unresectable advanced or metastatic melanoma [22]. The second review was undertaken to evaluate the efficacy and safety of the dabrafenib plus trametinib therapy compared to other first-line or second-line treatments for patients with unresectable or metastatic melanoma [23]. For both reviews, Embase $^{\circledR}, \quad$ MEDLINE ${ }^{\circledR} \quad$ including MEDLINE $^{\circledR}$ In-Process and other non-indexed citations),
Cochrane Central Trials Register, and key conferences (i.e., American Society of Clinical Oncology, European Society for Medical Oncology/European Cancer Organization, International Congress of the Society for Melanoma Research) were searched. Additionally, data reported in public assessment reports from the European Medicines Agency and Food and Drug Administration websites were searched, as were Health Technology Assessment (HTA) guidance documents published in English from selected HTA authorities (e.g., National Institute for Health and Care Excellence). The first review included randomized and non-randomized studies of dabrafenib, trametinib, DTIC, ipilimumab, vemurafenib, fotemustine, and temozolomide published through October 2012. The second review included randomized studies of dabrafenib plus trametinib, dabrafenib, trametinib, vemurafenib-cobimetinib combination, vemurafenib, ipilimumab, nivolumab, and pembrolizumab published through October 2015. For both reviews, screening and extraction was conducted by two independent reviewers. The first review identified 123 studies which met all inclusion criteria, of which 24 were randomized controlled trials in treatment-naïve patients [22]. The second review identified 12 randomized controlled trials of one or more of the treatment of interest in patients with unresectable or metastatic melanoma [23].

Combining the results of these two reviews, and focusing on trials of previously untreated patients receiving any one of the comparators approved in Canada at the time of the analysis, and which were required to estimate HRs for PFS and OS for the comparators versus dabrafenib plus trametinib, yielded seven studies, including the COMBI-d and BRF113220 (dabrafenib-trametinib versus dabrafenib), COMBI-v (dabrafenib-trametinib 
versus dabrafenib), BREAK-3 (dabrafenib versus DTIC), BRIM-3(vemurafenib versus DTIC), METRIC (ClinicalTrials.gov identifier: NCT01245062, trametinib versus chemotherapy), and CA184-024 (ClinicalTrials.gov identifier: NCT00324155, ipilimumab plus DTIC vs DTIC). HRs for PFS and OS were based on analyses of the most recent data cuts available from each trial at the time the evaluation was completed $[9,13,15,16,18,20,25]$. HRs for PFS and OS from the METRIC trial were based on analyses of the first-line subgroup. HRs for OS from the BRIM-3, BREAK-3, METRIC, and BRF113220 trials were based on Rank Preserving Structural Failure Time (RPSFT) analyses to adjust for crossover from control to active therapy [10, 14, 26, 27]. HRs for PFS and OS for ipilimumab were based on results of the CA184-024 trial [25]. Although this trial included both BRAF mutation-positive and BRAF wild-type patients, it was the only study available at the time of the analysis that reported both PFS and OS for ipilimumab as first-line treatment of metastatic melanoma. Also, although the approved dosage for ipilimumab is $3 \mathrm{mg} / \mathrm{kg} \mathrm{q} 3 \mathrm{w}$ as monotherapy, this trial compared ipilimumab $10 \mathrm{mg} / \mathrm{kg}$ plus DTIC versus DTIC. In the economic evaluation which this analysis informed, results for ipilimumab $10 \mathrm{mg} / \mathrm{kg}$ plus DTIC were used to estimate outcomes for patients receiving the approved dosage.

HRs were estimated by Bayesian network meta-analysis using WinBUGS 1.4.3@ (MRC Biostatistics Unit, Cambridge, UK) with input data taking the form of the log transformed HRs from each trial and the corresponding standard errors [28]. For each comparison, the log HRs for PFS and OS were estimated alternatively using multivariate network meta-analysis (in which treatment effects on PFS and OS were estimated simultaneously) and using traditional or univariate network meta-analysis (in which the analyses of PFS and OS were conducted separately). A multivariate network meta-analysis uses the correlation of the HRs for PFS and OS in the network to inform parameter estimation [29]. The use of this approach is reasonable given the well-established correlation between treatment effects on PFS and OS in metastatic melanoma [30].

Given the small number of trials with which to estimate random effects (in the analysis assuming no class effect for BRAF inhibitors, there was only one comparison for which there was more than one trial to estimate the random effects), a fixed effects model was employed. Model parameters were estimated using the WinBUGS software package (version 1.43) (Medical Research Council (MRC) Biostatistics Unit (BSU), Cambridge, UK). WinBUGS code used in the analysis are provided in the supplementary material.

Two sets of analyses were performed. In one, it was assumed that the effects of dabrafenib and vemurafenib on PFS and OS may be different ("no class-effect"), and separate HRs were estimated for dabrafenib and vemurafenib. In the other analysis, it was assumed that the effects of dabrafenib and vemurafenib on PFS and OS are the same ("class effect"). In this analysis, HRs for other comparators versus BRAF inhibitor monotherapy were calculated. This analysis permitted the combining of data on PFS and OS from the COMBI-d, COMBI-v, and BRF113220 trials in order to potentially improve the precision of the comparisons. Both analyses were conducted alternatively including and excluding the phase 2 BRF113220 trial. Multivariate network meta-analyses (in which the HRs for PFS and OS were estimated simultaneously to account for the correlation of these outcomes), were conducted both including and excluding trametinib and ipilimumab plus DTIC from 
the network, in order to account for the possibility that the correlation between treatment effects on OS and PFS differ by drug class.

The heterogeneity of effect sizes reported for all direct comparisons with multiple sources of direct evidence was evaluated using Cochrane's $\mathrm{Q}$ and the $I^{2}$ statistics. Consistency of direct and indirect comparisons within closed loops defined by the evidence network was confirmed by plotting the confidence intervals (CIs) of the difference in log HRs between direct and indirect comparisons.

This article does not contain any new studies with human or animal subjects performed by any of the authors.

\section{RESULTS}

Evidence network diagrams for PFS and OS for the analysis assuming no class-effect for BRAF inhibitors and the analysis assuming class-effect for BRAF inhibitor are shown in Fig. 1a, b, respectively. In the latter analysis, HRs for PFS and OS for the comparison of dabrafenib plus trametinib versus BRAF inhibitor monotherapy from COMB-v, COMBI-d and BRF113220 are pooled, as are the HRs for BRAF inhibitor monotherapy versus DTIC from BREAK-3 and BRIM-3.

Study design and patient characteristics are reported in Table 1. COMBI-v was the largest trial; BRF113220 was the smallest. Mean age ranged from 49 years (BRF113220, dabrafenib plus trametinib $1 \mathrm{mg}$ ) to 58 years (BRF113220, dabrafenib plus trametinib $2 \mathrm{mg}$ ). The percent male ranged from $49 \%$ (METRIC, DTIC) to $63 \%$ (BRF113220, dabrafenib plus trametinib $2 \mathrm{mg}$ ). The percent with Eastern Cooperative Oncology Group performance status $>0$ ranged from $25 \%$ (COMBI-d, dabrafenib plus trametinib) to $37 \%$
(BRF113220, dabrafenib). The percent with stage M1C at diagnosis ranged from 55\% (CA184-024, DTIC) to 70\% (BRF113220, dabrafenib plus trametinib $2 \mathrm{mg}$ ). The percent with elevated lactate dehydrogenase ranged from 30\% (BREAK-3, DTIC) to 58\% (BRIM-3, DTIC).

HRs used in the network meta-analyses are shown in Table 2. The HRs for PFS for the research arm versus the control arm were statistically significant for all trials. The HR for OS for the research arm versus the control arm was statistically significant for COMBI-v, COMBI-d, BRIM-3, and CA184-024. Note that the HRs for OS for BREAK-3, BRIM-3, METRIC, and BRF113220 are based on RPSFT analyses that adjust for crossover from control to active therapy. The 95\% CIs for these HRs are therefore relatively wide compared to those in other trials.

Results of the network meta-analysis on HRs for PFS and OS for the analysis assuming no class-effect for BRAF inhibitors are shown in Table 3 and in Fig. 2. All HRs (95\% CrI) for PFS significantly favored dabrafenib plus trametinib: $0.23(0.18-0.29)$ versus DTIC, 0.32 (0.24-0.42) versus ipilimumab plus DTIC, 0.52 (0.32-0.83) versus trametinib, 0.57 (0.48-0.69) versus vemurafenib, and $0.59(0.50-0.71)$ versus dabrafenib. All HRs (95\% CrI) for OS also significantly favored dabrafenib plus trametinib: $0.41(0.29-0.56)$ versus DTIC, 0.52 (0.38-0.71) versus ipilimumab plus DTIC, 0.68 (0.47-0.95) versus trametinib, 0.69 (0.57-0.84) versus vemurafenib, and $0.72(0.60-0.85)$ versus dabrafenib. Results were similarly in favor of dabrafenib plus trametinib when the Phase II BRF113220 trial was excluded from the network. Excluding ipilimumab plus DTIC and trametinib from the network had little impact on the HRs for the other therapies. When HRs 


\section{A Analysis assuming no class-effect for BRAF inhibitors}

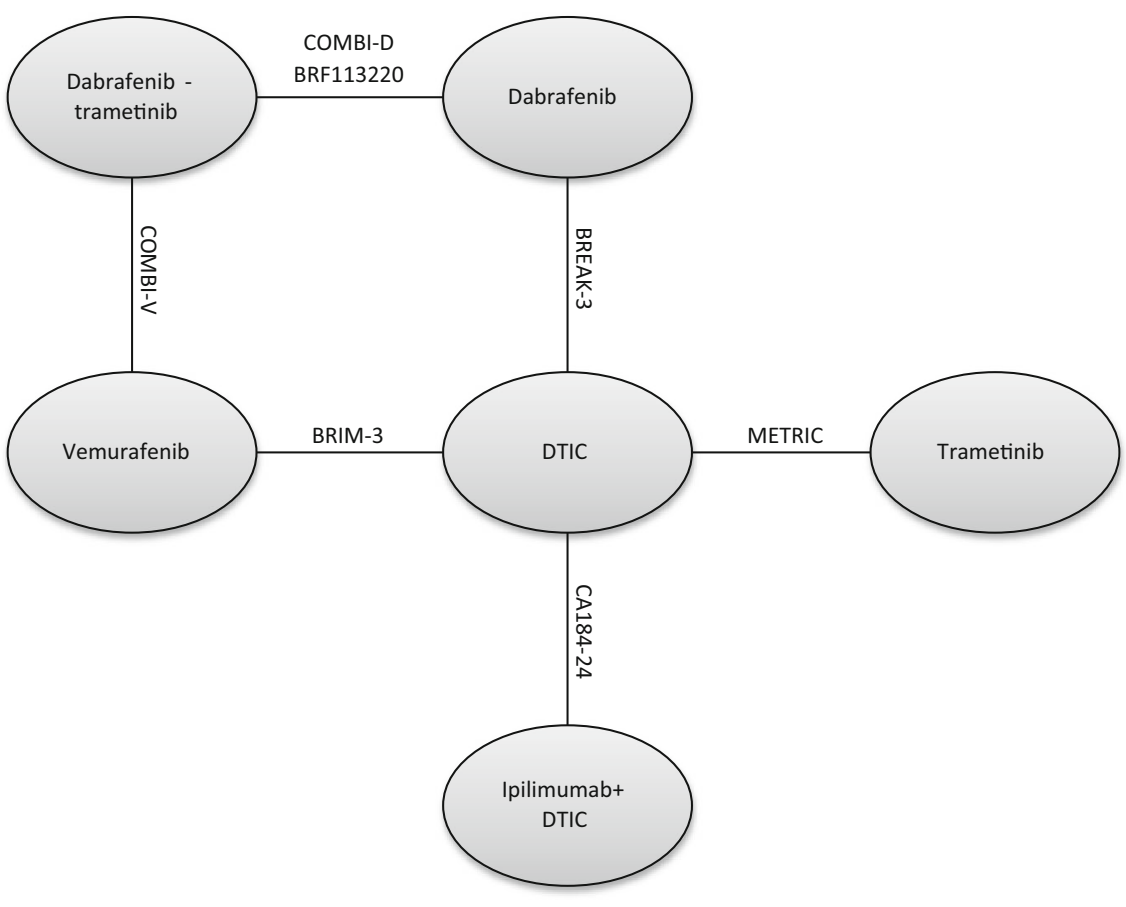

\section{B Assuming class-effect for BRAF-inhibitors}

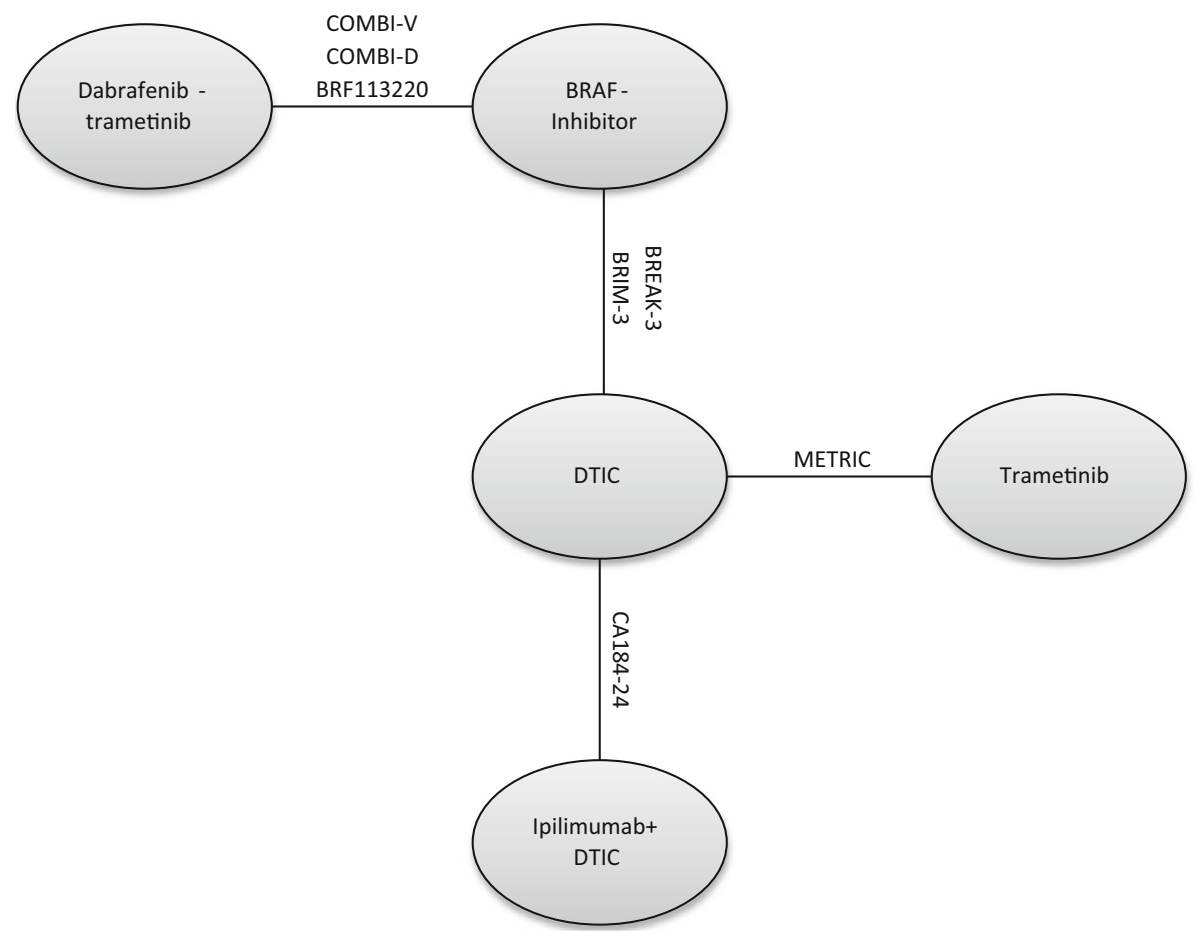

Fig. 1 Evidence network for network meta-analyses of hazard ratios for progression-free survival and overall survival. a Analysis assuming no class-effect for BRAF inhibitors, $\mathbf{b}$ assuming class-effect for BRAF inhibitors. DTIC dacarbazine 


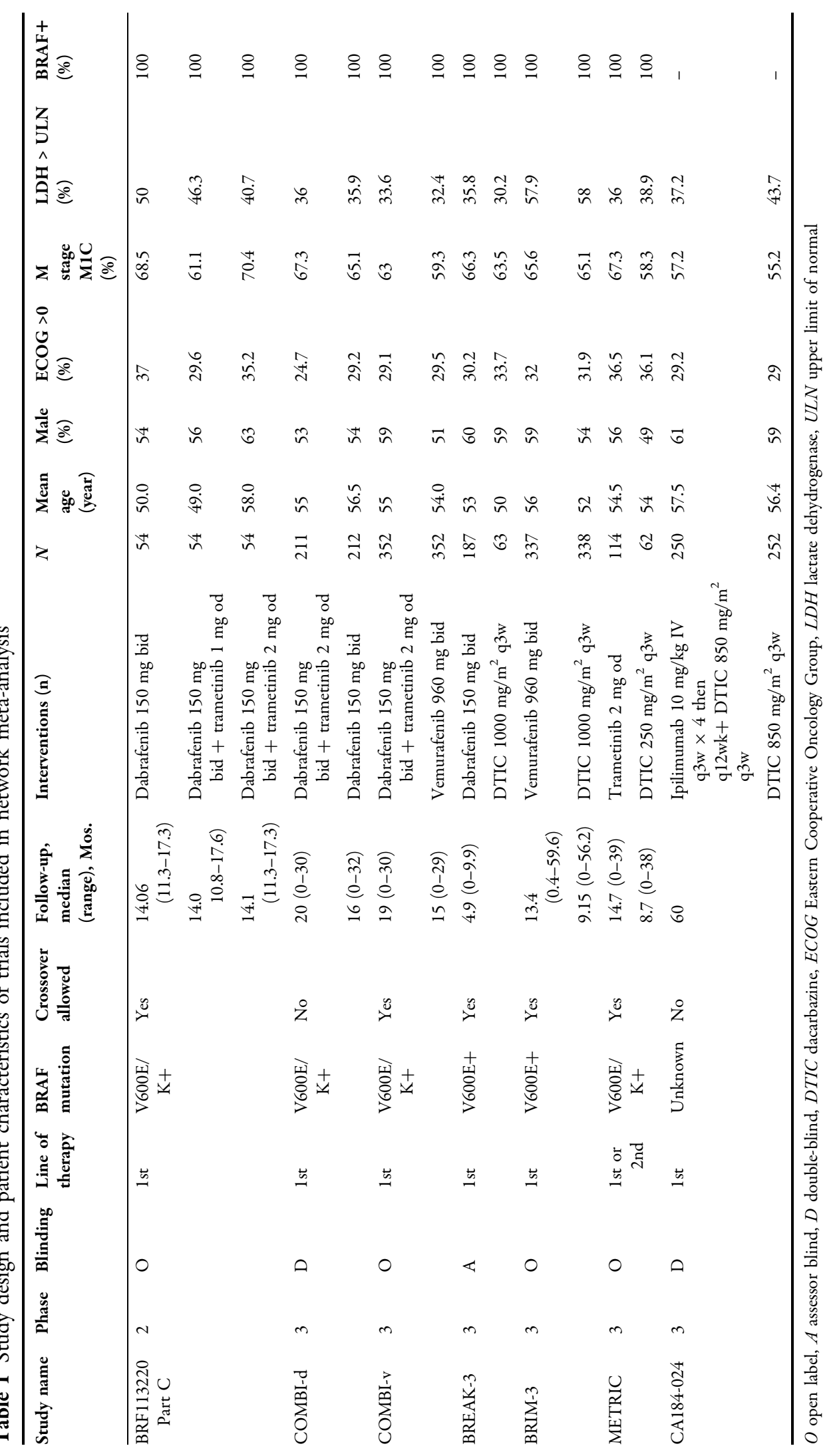


Table 2 HRs for PFS and OS used in network meta-analysis

\begin{tabular}{|c|c|c|c|c|c|c|c|c|c|}
\hline Study & Research & Control & $\begin{array}{l}\text { Data } \\
\text { cut-off }\end{array}$ & $\begin{array}{l}\mathrm{N} \\
\text { research }\end{array}$ & $\begin{array}{l}\mathrm{N} \\
\text { control }\end{array}$ & RPSFT & HR & \multicolumn{2}{|c|}{$95 \% \mathrm{CI}$} \\
\hline \multicolumn{10}{|l|}{ PFS } \\
\hline COMBI-v & Dabrafenib + trametinib & Vemurafenib & 17-Apr-14 & 352 & 352 & $\mathrm{n} / \mathrm{a}$ & 0.56 & 0.46 & 0.69 \\
\hline COMBI-d & Dabrafenib + trametinib & Dabrafenib & 12-Jan-15 & 211 & 212 & $\mathrm{n} / \mathrm{a}$ & 0.67 & 0.53 & 0.84 \\
\hline BRF113220 & Dabrafenib + trametinib & Dabrafenib & 15-Jan-15 & 54 & 54 & $\mathrm{n} / \mathrm{a}$ & 0.41 & 0.27 & 0.64 \\
\hline BREAK-3 & Dabrafenib & DTIC & 25-Jun-12 & 187 & 63 & $\mathrm{n} / \mathrm{a}$ & 0.37 & 0.23 & 0.57 \\
\hline BRIM- $3^{a}$ & Vemurafenib & DTIC & $12-F e b-12$ & 337 & 338 & $\mathrm{n} / \mathrm{a}$ & 0.38 & 0.32 & 0.46 \\
\hline METRIC $^{b}$ & Trametinib & DTIC & 26 Oct 11 & 114 & 62 & $\mathrm{n} / \mathrm{a}$ & 0.44 & 0.28 & 0.69 \\
\hline CA184-24 & Ipilimumab + DTIC & DTIC & $\mathrm{n} / \mathrm{r}$ & 247 & 251 & $\mathrm{n} / \mathrm{a}$ & 0.76 & 0.63 & 0.93 \\
\hline \multicolumn{10}{|l|}{ OS } \\
\hline COMBI-v & Dabrafenib + trametinib & Vemurafenib & 17-Apr-14 & 352 & 352 & No & 0.69 & 0.53 & 0.89 \\
\hline COMBI-d & Dabrafenib + trametinib & Dabrafenib & 12-Jan-15 & 211 & 212 & No & 0.71 & 0.55 & 0.92 \\
\hline BRF113220 & Dabrafenib + trametinib & Dabrafenib & 29-Mar-13 & 54 & 54 & Yes & 0.47 & 0.13 & 1.66 \\
\hline BREAK-3 & Dabrafenib & DTIC & 18-Dec-12 & 187 & 63 & Yes & 0.55 & 0.21 & 1.43 \\
\hline BRIM-3 & Vemurafenib & DTIC & $12-F e b-12$ & 337 & 338 & Yes & 0.64 & 0.47 & 0.88 \\
\hline METRIC $^{a}$ & Trametinib & DTIC & 16-May-13 & 114 & 62 & Yes & 0.44 & 0.20 & 1.00 \\
\hline CA184-024 & Ipilimumab + DTIC & DTIC & $\mathrm{n} / \mathrm{r}$ & 247 & 251 & No & 0.72 & 0.59 & 0.87 \\
\hline
\end{tabular}

$H R$ hazard ratio, PFS progression-free survival, $O S$ overall survival, DTIC dacarbazine, RPSFT rank preserving structural failure time

a The reported confidence interval for the RPSFT adjusted HR for OS for BRIM-3 (HR = 0.64, 95\% CI 0.53-0.78) implied a lower $p$ value than that from the intent-to-treat analysis (HR $=0.76,95 \%$ CI $0.63-0.93$ ). Since the RPSFT method does not increase statistical power, the reported confidence interval was assumed to be erroneous. The confidence interval used in the analysis was obtained by solving for the standard error on the $\log (\mathrm{HR})$ which yielded the same $p$ value as the corresponding intent-to-treat analysis

b First-line subgroup of primary efficacy population

for PFS and OS were estimated separately using univariate network meta-analysis (i.e., without accounting for the correlation of treatment effects on PFS and OS), the beneficial effects on OS of dabrafenib plus trametinib versus ipilimumab plus DTIC and dabrafenib plus trametinib versus trametinib were reduced compared with estimates from the multivariate analysis in which the HRs for PFS and OS were estimated simultaneously (compared with trametinib, $\mathrm{HR}=0.68$ for multivariate analysis versus 0.98 for univariate analysis; compared with ipilimumab plus DTIC, $\mathrm{HR}=0.52$ for multivariate analysis versus 0.60 for univariate analysis).

Results of the network meta-analysis on HRs for PFS and OS for the analysis assuming a class-effect for BRAF inhibitors are shown in Fig. 3. These results were generally similar to those for the analysis in which separate HRs were estimated for dabrafenib and vemurafenib monotherapy. When all trials and treatments 


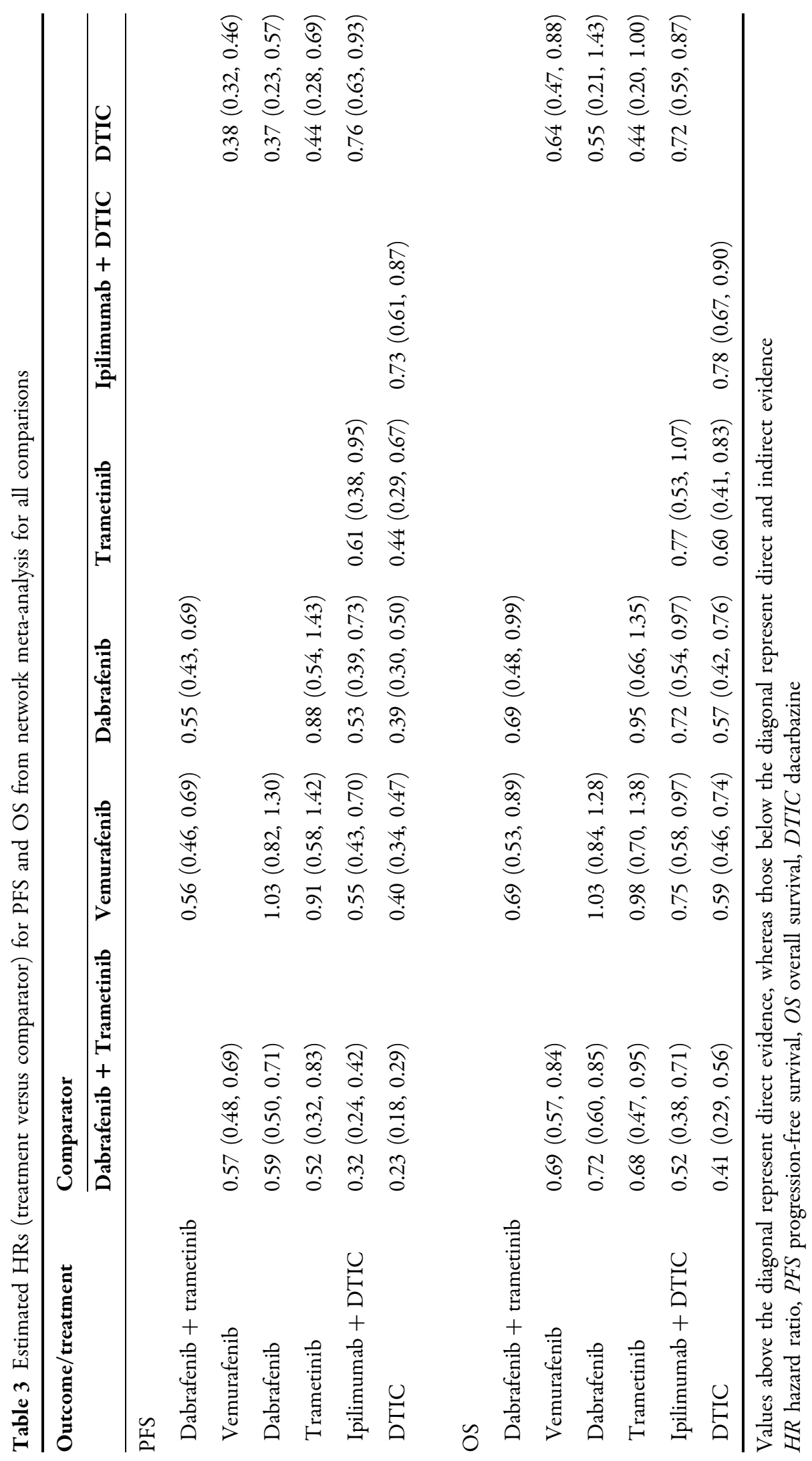



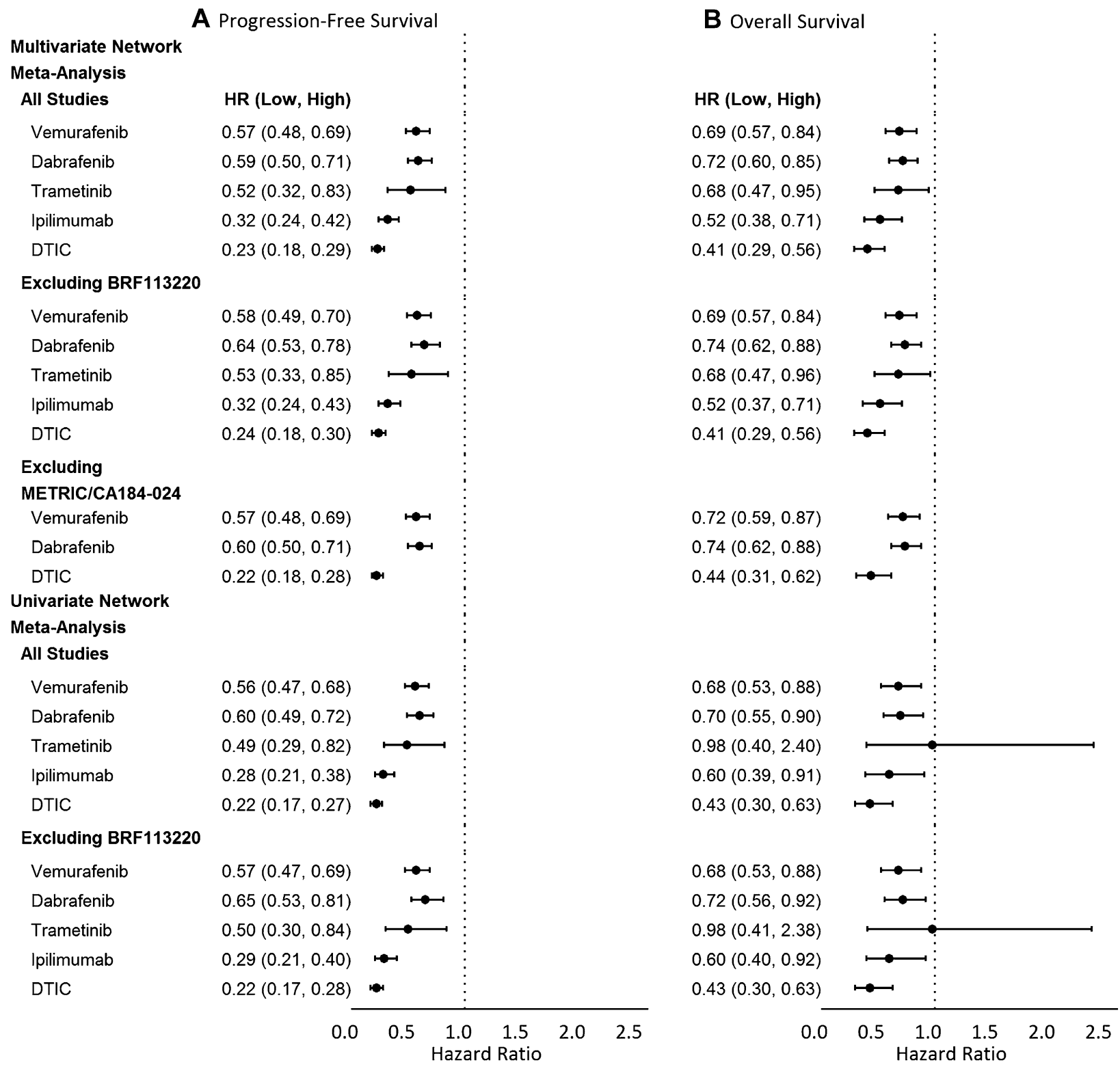

Fig. 2 Results of network meta-analyses of HRs for progression-free survival and overall survival for dabrafenib plus trametinib versus other treatments. HR hazard ratio, DTIC dacarbazine

were included, the HRs for PFS and OS for dabrafenib plus trametinib were significantly less than 1.0 for all comparisons. The HRs and 95\% CrI for dabrafenib plus trametinib versus trametinib, ipilimumab plus DTIC, and DTIC were similar to those obtained in the analysis in which no class-effect was assumed.
For all comparisons of OS based on multiple sources of direct evidence, the $I^{2}$ statistic was estimated to be $0 \%$, indicating no identifiable heterogeneity. For PFS, significant heterogeneity of treatment effects was observed only for the PFS HR for the comparison of dabrafenib plus trametinib versus dabrafenib based on COMBI-d 

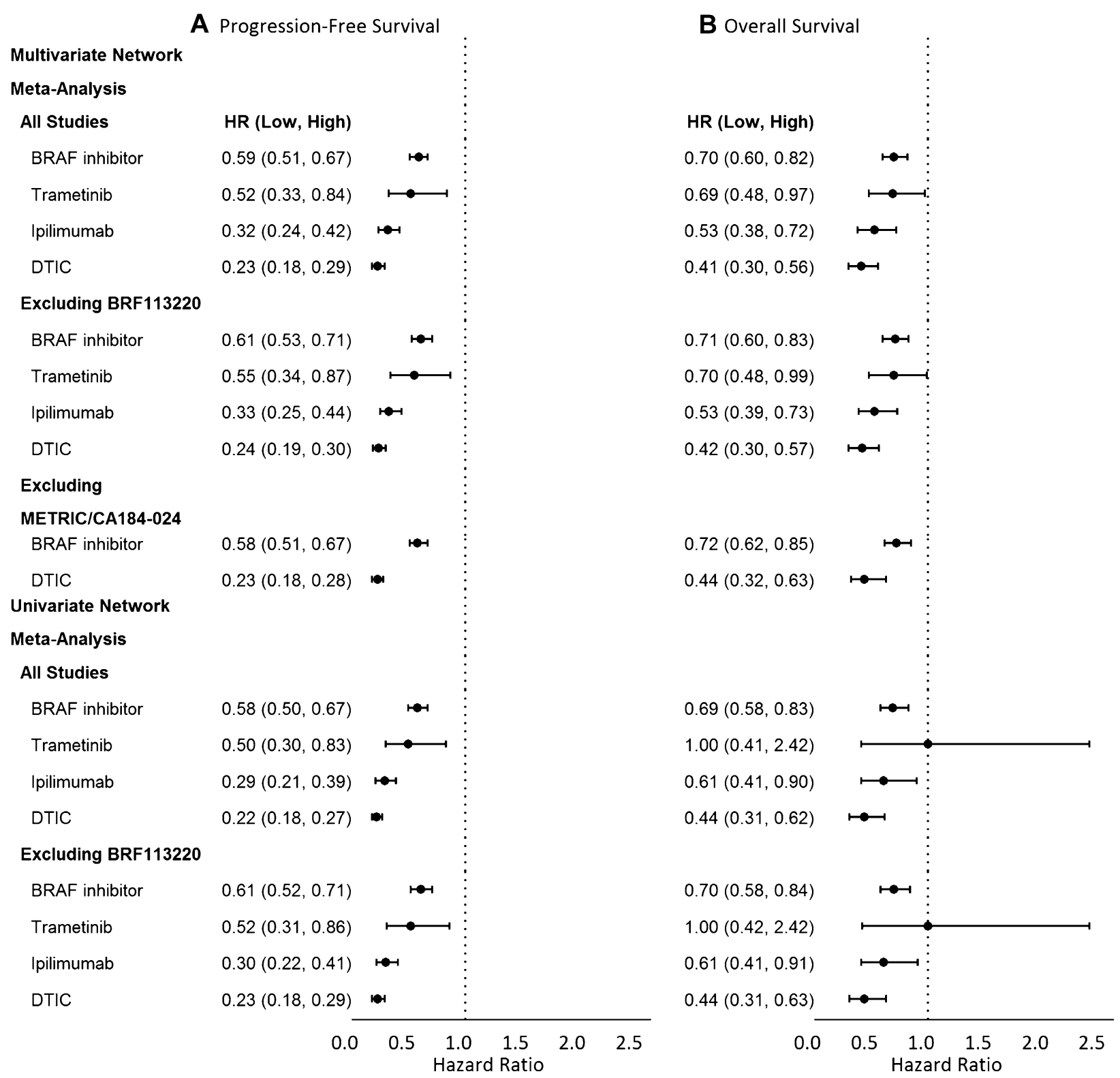

Fig. 3 Results of network meta-analyses of HRs for progression-free survival and overall survival for dabrafenib plus trametinib versus other treatments assuming class effect for BRAF inhibitors. HR hazard ratio, DTIC dacarbazine

and BRF113220 $(Q=3.873, \quad p=0.049$, $\left.I^{2}=74.2 \%\right)$. When assuming a class effect, COMBI-V is included in the comparison, heterogeneity remains elevated, but drops below the threshold for significance $\left(Q=4.338, \quad p=0.114, \quad I^{2}=53.9 \%\right) . \quad$ No heterogeneity was identified in the comparison of BRAF monotherapies and DTIC based on
BREAK-3 and BRIM-3 $(Q=0.011, p=0.915$, $\left.I^{2}=0.0 \%\right)$. There was no evidence of inconsistency of direct and indirect estimates of treatment effects within the closed loop formed by the BREAK-3, BRIM-3, COMBI-v, and COMBI-d studies, as the CIs for the differences of $\log$ HRs between direct and indirect effects spanned zero for both PFS and OS. 


\section{DISCUSSION}

This study was a network meta-analysis of HRs for PFS and OS of dabrafenib plus trametinib versus other first-line treatments of BRAF mutation-positive metastatic melanoma that was conducted to inform an economic evaluation of dabrafenib plus trametinib as first-line treatment of metastatic melanoma in Canada. Results of this analysis suggest that dabrafenib plus trametinib yields improved PFS and OS compared to other first-line treatment for BRAF mutation-positive metastatic melanoma. The results for dabrafenib-trametinib were more favorable compared with trametinib and ipilimumab plus DTIC when HRs for PFS and OS were estimated using multivariate network meta-analysis to account for the correlation of treatment effects on PFS and OS than when they were estimated separately using traditional univariate network meta-analysis. Results were generally similar when it was assumed there was a class effect on PFS and OS for BRAF inhibitor monotherapy compared with analyses in which no such assumption was made. Results also were similar when the phase 2 BRF113220 trial was included or excluded.

A targeted search identified several network meta-analyses of treatments for metastatic melanoma [22, 31-34]. Two of these articles focused on comparisons of chemotherapies and interferon [32, 33]. One study focused on comparisons of ipilimumab versus immunotherapies, chemotherapies, and biochemotherapies, but did not consider BRAF or MEK inhibitors [34]. Srivastava and colleagues conducted a systematic review and indirect treatment comparison of dabrafenib and trametinib as monotherapy versus other treatments in previously untreated metastatic melanoma patients; this study did not include
BRAF-MEK combination therapy, however [22]. Only one study was identified that included dabrafenib plus trametinib [31]. This study by Mai and colleagues compared BRAF-MEK inhibition with BRAF inhibitor monotherapy, MEK inhibitor monotherapy, BRAF inhibitor plus chemotherapy, and MEK inhibitor plus chemotherapy [31]. The study did not consider ipilimumab. The authors reported that combined BRAF-MEK inhibition significantly prolonged PFS compared with BRAF inhibition alone $(\mathrm{HR}=0.58,95 \% \mathrm{CI} 0.51-0.67, p<0.0001)$ and MEK inhibition alone $(\mathrm{HR}=0.29,95 \% \mathrm{CI}$ $0.22-0.37, p<0.0001)$. Similar results were obtained for OS for BRAF-MEK inhibition versus BRAF inhibition, $(\mathrm{HR}=0.67,95 \% \mathrm{CI}$ $0.56-0.81, p<0.0001)$ and for BRAF-MEK inhibition versus MEK inhibition $(\mathrm{HR}=0.48$, 95\% CI $0.36-0.65, p<0.0001)$. These results are qualitatively similar to those reported herein.

Limitations of this study should be noted. Perhaps most importantly, this analysis did not consider new immunotherapies such as nivolumab and pembrolizumab, or the combination of vemurafenib plus the MEK inhibitor, cobimetinib, as these therapies were not approved in Canada at the time this analysis was conducted and therefore were not required in the economic evaluation for which this network meta-analysis was performed. Future network meta-analyses of therapies for metastatic melanoma should include these novel treatments if feasible. It should be noted, however, that the robustness of any such analyses will depend on the availability of efficacy data on both PFS and OS in patients with BRAF mutations positive disease.

Differences in patients, study design, and duration of follow-up may have affected treatment effects thus violating the similarity assumption and confounding the comparisons. For example, the COMBI-d and CA184-024 
trials were double-blind, whereas the other trials in the network were open label. The CA184-024 trial included both BRAF mutation-positive and wild-type patients. These factors may modify treatment effects and bias the comparisons. Given the small number of studies it was not possible to assess the impact of these differences on results.

This study used HRs as the measure of treatment effect. Use of HRs requires an assumption of proportional hazards which may not hold across all studies. Ouwens et al. [35] have described an approach for conducting a network meta-analysis of parametric survival distributions that may address the limitations of conducting the analysis based on HRs. However, this approach requires additional assumptions regarding the similarity of underlying parametric survival distributions across trials that may not hold. Nevertheless, this is a potentially important area for future research.

The approved dosage for ipilimumab in North America and Europe is $3 \mathrm{mg} / \mathrm{kg}$ once every three weeks. However, the systematic literature reviews did not identify any controlled trials of ipilimumab monotherapy versus DTIC at this dosage. Accordingly, the HRs for PFS and OS for ipilimumab $10 \mathrm{mg} / \mathrm{kg}$ plus DTIC versus DTIC from the CA184-024 trial were used in the evidence network. The use of the results of this analysis to estimate the relative efficacy of ipilimumab monotherapy $3 \mathrm{mg} / \mathrm{kg}$ versus other comparators requires the assumptions (1) that there is no difference in treatment effect on PFS and OS for the 10 and $3 \mathrm{mg} / \mathrm{kg}$ doses of ipilimumab and (2) that the addition of DTIC to ipilimumab has no impact on treatment effects on PFS or OS. Data on the impact of the addition of DTIC to ipilimumab on outcomes are limited and inconclusive. In the MDX010-08 trial, an open label phase 2 study of ipilimumab $3 \mathrm{mg} / \mathrm{kg}$ plus DTIC versus ipilimumab $3 \mathrm{mg} / \mathrm{kg}$ in 76 previously treated patients with metastatic melanoma [36], the addition of DTIC to ipilimumab was associated with nominally improved response, PFS, and OS. These results were not conclusive, however, given the small size of the trial. Median PFS was 99 and 85 days in the ipilimumab plus DTIC and ipilimumab groups, respectively. Median OS was 14.3 versus 11.4 months for the ipilimumab plus DTIC and ipilimumab groups, respectively.

Regarding the assumption that the efficacy of ipilimumab $10 \mathrm{mg} / \mathrm{kg}$ is equivalent to $3 \mathrm{mg} /$ $\mathrm{kg}$, the CA184-004 trial compared ipilimumab 3 versus $10 \mathrm{mg} / \mathrm{kg}$ in a mixed population of 101 melanoma patients with and without prior treatment. There was no evidence of improved PFS or OS with 10 versus $3 \mathrm{mg} / \mathrm{kg}$ in this trial $[37,38]$. The CA184-022 trial compared 0.3, 3, and $10 \mathrm{mg} / \mathrm{kg}$ in 214 previously treated patients with melanoma [39, 40]. This study demonstrated a statistically significant dose-response relationship on best overall response rates. Median OS and 1- and 2-year survival rates were nominally improved with ipilimumab $10 \mathrm{mg} / \mathrm{kg}$ compared with 3 and $0.3 \mathrm{mg} / \mathrm{kg}$. These results are suggestive that adding DTIC to ipilimumab may provide some benefit and that $10 \mathrm{mg} / \mathrm{kg}$ may improve outcomes versus $3 \mathrm{mg} / \mathrm{kg}$. Accordingly, the use of the CA184-024 trial to estimate the relative effectiveness of ipilimumab $3 \mathrm{mg} / \mathrm{kg}$ versus DTIC may bias estimates of the relative effectiveness in favor of ipilimumab and versus BRAF and MEK inhibitors.

Trials of BRAF and MEK inhibitors focused on patients with BRAF mutations, whereas the CA184-024 trial focused on a mix of patients with BRAF mutations and wild-type (WT) disease. Known BRAF mutation status was not required for inclusion in the CA184-024 trial 
and data on PFS and OS for subgroups of patients defined on BRAF mutation status have not been reported. Evidence suggests that BRAF-mutant melanoma is biologically distinct from BRAF WT disease [41-44]. Also, numerous studies have shown that BRAF-mutant melanoma is associated with more aggressive disease and worse patient outcomes [41-47]. Because data on treatment effects for ipilimumab versus DTIC by BRAF mutation status are unavailable, it was not possible to assess potential effect modification from this factor.

To the best of our knowledge, this is the first study to use both univariate and multivariate network meta-analysis to estimate HRs for PFS and OS. Multivariate network meta-analysis of HRs for PFS and OS accounts for the well-established correlation of treatment effects on PFS and OS. In the COMBI-d, COMBI-v, BREAK-3, and BRIM-3 trials, treatment effects on PFS were greater than treatment effects on OS. In the METRIC trial, the estimated treatment effect of trametinib on OS was equal to that on PFS $(\mathrm{HR}=0.44$ for both). In the CA184-024 trial, the estimated treatment effect of ipilimumab plus DTIC on OS was greater than that on PFS (HR $=0.76$ for PFS and $\mathrm{HR}=0.72$ for OS). As a consequence, the multivariate network meta-analysis attenuated the estimated treatment effects on OS for trametinib and ipilimumab plus DTIC. For example, the HR for OS for trametinib versus DTIC observed in METRIC was 0.44 (95\% CI 0.20-1.00), whereas that obtained from the multivariate network meta-analysis was 0.60 (95\% CI 0.41-0.83). Similarly, the HR for OS for ipilimumab plus DTIC versus DTIC observed in CA184-024 was 0.72 (95\% CI 0.59-0.87), whereas that obtained from the multivariate network meta-analysis was $0.78 \quad(95 \%$ CI 0.67-0.90). The results of these analyses may be biased if there are unique attributes of trametinib and ipilimumab that would result in differential relative effects on OS versus PFS compared with the other therapies. For trametinib, we know of no reason to believe that this would be the case; rather, it is more likely a consequence of the small number of subjects in the first-line subgroup of the METRIC trial and the imprecision in the RPSFT method for adjustment for crossover. For ipilimumab, however, it is possible that the immune-related effects may be delayed relative to the cytotoxic effects of BRAF and MEK inhibitors, which would explain a relatively large effect on OS versus that on PFS. The analysis based on the multivariate network meta-analysis may have biased the results in favor of the combination and against ipilimumab plus DTIC therefore should be interpreted cautiously.

\section{CONCLUSION}

In conclusion, this network meta-analysis demonstrates improved PFS and OS with dabrafenib plus trametinib versus dabrafenib, trametinib, vemurafenib, ipilimumab, and DTIC as first-line therapy for patients with BRAF mutation-positive metastatic melanoma. Future research should be conducted which includes other novel treatments, if feasible, and based on network meta-analysis of survival distributions rather than HRs to account for non-proportionality of hazards.

\section{ACKNOWLEDGMENTS}

Funding for this research was provided by Novartis Pharmaceuticals. No funding or sponsorship was received for article page charges. All authors had full access to all of 
the data in this study and take complete responsibility for the integrity of the data and accuracy of the data analysis. All named authors meet the International Committee of Medical Journal Editors (ICMJE) criteria for authorship for this manuscript, take responsibility for the integrity of the work as a whole, and have given final approval for the version to be published.

Disclosures. Thomas Delea received research funding and consulting from GlaxoSmithKline and Novartis. Jordan Amdahl received research funding and consulting from GlaxoSmithKline and Novartis. Lei Chen is employed by Novartis and owns stock or stock options in Novartis.

Compliance with ethics guidelines. This article does not contain any new studies with human or animal subjects performed by any of the authors.

Open Access. This article is distributed under the terms of the Creative Commons Attribution-NonCommercial 4.0 International License (http://creativecommons.org/licenses/ by-nc/4.0/), which permits any noncommercial use, distribution, and reproduction in any medium, provided you give appropriate credit to the original author(s) and the source, provide a link to the Creative Commons license, and indicate if changes were made.

\section{REFERENCES}

1. Hodi FS, O'Day SJ, McDermott DF, Weber RW, Sosman JA, Haanen JB, et al. Improved survival with ipilimumab in patients with metastatic melanoma. N Engl J Med. 2010;363:711-23. doi:10.1056/NEJMoa1003466.

2. Schadendorf D, Hodi FS, Robert C, Weber JS, Margolin K, Hamid O, et al. Pooled analysis of long-term survival data from phase II and phase III trials of ipilimumab in unresectable or metastatic melanoma. J Clin Oncol. 2015;33:1889-94. doi:10. 1200/JCO.2014.56.2736.

3. Robert C, Thomas L, Bondarenko I, O’Day S, Weber $\mathrm{J}$, Garbe $\mathrm{C}$, et al. Ipilimumab plus dacarbazine for previously untreated metastatic melanoma. N Engl J Med. 2011;364:2517-26. doi:10.1056/ NEJMoa1104621.

4. Robert C, Schachter J, Long GV, Arance A, Grob JJ, Mortier L, et al. Pembrolizumab versus ipilimumab in advanced melanoma. $\mathrm{N}$ Engl J Med. 2015;372:2521-32. doi:10.1056/NEJMoa1503093.

5. Postow MA, Chesney J, Pavlick AC, Robert C, Grossmann K, McDermott D, et al. Nivolumab and ipilimumab versus ipilimumab in untreated melanoma. N Engl J Med. 2015;372:2006-17. doi:10.1056/NEJMoa1414428.

6. Larkin J, Hodi FS, Wolchok JD. Combined nivolumab and ipilimumab or monotherapy in untreated melanoma. N Engl J Med. 2015;373:1270-1. doi:10.1056/NEJMc1509660.

7. Wang AX, Qi XY. Targeting RAS/RAF/MEK/ERK signaling in metastatic melanoma. IUBMB Life. 2013;65:748-58. doi:10.1002/iub.1193.

8. Chapman PB, Hauschild A, Robert C, Haanen JB, Ascierto P, Larkin J, et al. Improved survival with vemurafenib in melanoma with BRAF V600E mutation. N Engl J Med. 2011;364:2507-16. doi:10.1056/NEJMoa1103782.

9. McArthur GA, Chapman PB, Robert C, Larkin J, Haanen JB, Dummer R, et al. Safety and efficacy of vemurafenib in BRAF(V600E) and BRAF(V600K) mutation-positive melanoma (BRIM-3): extended follow-up of a phase 3, randomised, open-label study. Lancet Oncol. 2014;15:323-32. doi:10.1016/ S1470-2045(14)70012-9.

10. Beale S, Dickson R, Bagust A, Blundell M, Dundar Y, Boland A, et al. Vemurafenib for the treatment of locally advanced or metastatic BRAF V600 mutation-positive malignant melanoma: a NICE single technology appraisal. Pharmacoeconomics. 2013;31:1121-9. doi:10.1007/s40273-013-0094-x.

11. Hauschild A, Grob JJ, Demidov LV, Jouary T, Gutzmer R, Millward $M$, et al. Dabrafenib in BRAF-mutated metastatic melanoma: a multicentre, open-label, phase 3 randomised controlled trial. Lancet. 2012;380:358-65. doi:10. 1016/S0140-6736(12)60868-X.

12. Hauschild A, Grob JJ, Demidov LV, Jouary T, Gutzmer R, Millward $M$, et al. An update on overall survival (OS) and follow-on therapies in BREAK-3, a phase III, randomized trial: dabrafenib (D) versus dacarbazine (DTIC) in patients (pts) with 
BRAF V600E mutation-positive metastatic melanoma (MM). Presented at the European Society for Medical Oncology Annual Meeting, 29 September 2014. 2014. http://oncologypro.esmo. org/Meeting-Resources/ESMO-2014/Melanoma-andOther-Skin-Tumours/An-update-on-overall-survivalOS-and-follow-on-therapies-in-BREAK-3-a-Phase-IIIrandomized-trial-dabrafenib-D-vs.-dacarbazine-DTICin-patients-pts-with-BRAF-V600E-mutation-positivemetastatic-melanoma-MM. Accessed 16 August 2016.

13. Grob J-J, Demidov LV, Jouary T, Gutzmer R, Millward M, Rutkowski $\mathrm{P}$, et al. A landmark analysis of 3-year overall survival (OS) and follow-on therapies in BREAK-3, a phase III, randomized trial: dabrafenib versus dacarbazine (DTIC) in patients (pts) with BRAF V600E mutation-positive metastatic melanoma. 2014 Society for Melanoma Research Annual Meeting. Zurich, Switzerland. 2014.

14. Latimer NR, Abrams KR, Amonkar MM, Stapelkamp C, Swann RS. Adjusting for the confounding effects of treatment switching-the BREAK-3 trial: dabrafenib versus dacarbazine. Oncologist. 2015;20:798-805. doi:10.1634/theoncologist.20140429.

15. Flaherty KT, Robert C, Hersey P, Nathan P, Garbe C, Milhem $\mathrm{M}$, et al. Improved survival with MEK inhibition in BRAF-mutated melanoma. N Engl J Med. 2012;367:107-14. doi:10.1056/ NEJMoa1203421.

16. Robert C, Karaszewska B, Schachter J, Rutkowski P, Mackiewicz A, Stroiakovski D, et al. Improved overall survival in melanoma with combined dabrafenib and trametinib. $\mathrm{N}$ Engl J Med. 2015;372:30-9. doi:10.1056/NEJMoa1412690.

17. Long GV, Stroyakovskiy D, Gogas H, Levchenko E, de Braud F, Larkin J, et al. Combined BRAF and MEK inhibition versus BRAF inhibition alone in melanoma. N Engl J Med. 2014;371:1877-88. doi:10.1056/NEJMoa1406037.

18. Long GV, Stroyakovskiy D, Gogas H, Levchenko E, de Braud F, Larkin J, et al. Dabrafenib and trametinib versus dabrafenib and placebo for Val600 BRAF-mutant melanoma: a multicentre, double-blind, phase 3 randomised controlled trial. Lancet. 2015;386:444-51. doi:10.1016/S01406736(15)60898-4.

19. Flaherty KT, Infante JR, Daud A, Gonzalez R, Kefford RF, Sosman J, et al. Combined BRAF and MEK inhibition in melanoma with BRAF V600 mutations. N Engl J Med. 2012;367:1694-703. doi:10.1056/NEJMoa1210093.
20. Flaherty K, Daud A, Weber J, Sosman J, Kim K, Gonzalez R, et al. Updated overall survival for BRF113220: a phase 1-2 study of dabrafenib alone versus combined dabrafenib and trametinib in patients with BRAF V600 mutation-positive metastatic melanoma. J Clin Oncol. 2014;32:Abstract 9010. http://meetinglibrary.asco. org/content/134903-144. Accessed 16 August 2016.

21. Daud A, Weber J, Sosman J, Kim K, Gonzalez R, Hamid $O$, et al. Updated overall survival for BRF113220: a phase 1-2 study of dabrafenib alone vs combined dabrafenib and trametinib in patients with BRAF V600 mutation-positive metastatic melanoma. J Clin Oncol. 2015;33:Abstract 9036. http://meetinglibrary.asco.org/content/147629156. Accessed 16 August 2016.

22. Srivastava K, Amonkar MM, Ahuja A, Stapelkamp C, Swann S, Casey M, et al. Systematic review and indirect treatment comparison of dabrafenib and trametinib versus other treatments used in previously untreated metastatic melanoma patients. J Clin Exp Dermatol Res. 2015;6:13. doi:10.4172/2155-9554.1000294.

23. National Institute for Health and Care Excellence. Melanoma (BRAF V600E mutation positive, unresectable, metastatic)—dabrafenib and trametinib [ID661]. 2016. https://www.nice.org. uk/guidance/indevelopment/gid-tag365. Accessed 16 August 2016.

24. Liberati A, Altman DG, Tetzlaff J, Mulrow C, Gotzsche PC, Ioannidis JP, et al. The PRISMA statement for reporting systematic reviews and meta-analyses of studies that evaluate healthcare interventions: explanation and elaboration. BMJ. 2009;339:b2700. doi:10.1136/bmj.b2700.

25. Maio M, Grob JJ, Aamdal S, Bondarenko I, Robert C, Thomas L, et al. Five-year survival rates for treatment-naive patients with advanced melanoma who received ipilimumab plus dacarbazine in a phase III trial. J Clin Oncol. 2015;33:1191-6. doi:10.1200/JCO.2014.56.6018.

26. Latimer NR, Amonkar MM, Stapelkamp C, Sun P. Adjusting for confounding effects of treatment switching in a randomized phase II study of dabrafenib plus trametinib in BRAF V600+ metastatic melanoma. Melanoma Res. 2015;25:528-36. doi:10. 1097/CMR.0000000000000193.

27. Latimer NR, Bell H, Abrams KR, Amonkar MM, Casey M. Adjusting for treatment switching in the METRIC study shows further improved overall survival with trametinib compared with chemotherapy. Cancer Med. 2016;. doi:10.1002/ cam4.643. 
28. Dias S, Sutton AJ, Ades AE, Welton NJ. Evidence synthesis for decision making 2: a generalized linear modeling framework for pairwise and network meta-analysis of randomized controlled trials. Med Decis Making. 2013;33:607-17. doi:10.1177/ $0272989 \times 12458724$.

29. Achana FA, Cooper NJ, Bujkiewicz S, Hubbard SJ, Kendrick D, Jones DR, et al. Network meta-analysis of multiple outcome measures accounting for borrowing of information across outcomes. BMC Med Res Methodol. 2014;14:92. doi:10.1186/14712288-14-92.

30. Flaherty KT, Hennig $M$, Lee SJ, Ascierto PA, Dummer R, Eggermont AM, et al. Surrogate endpoints for overall survival in metastatic melanoma: a meta-analysis of randomised controlled trials. Lancet Oncol. 2014;15:297-304. doi:10.1016/S1470-2045(14)70007-5.

31. Mai R, Zhou S, Zhong W, Rong S, Cong Z, Li Y, et al. Therapeutic efficacy of combined BRAF and MEK inhibition in metastatic melanoma: a comprehensive network meta-analysis of randomized controlled trials. Oncotarget. 2015;6:28502-12. doi:10.18632/oncotarget. 4375 .

32. Cope S, Jansen JP. Quantitative summaries of treatment effect estimates obtained with network meta-analysis of survival curves to inform decision-making. BMC Med Res Methodol. 2013;13:147. doi:10.1186/1471-2288-13-147.

33. Jansen JP, Cope S. Meta-regression models to address heterogeneity and inconsistency in network meta-analysis of survival outcomes. BMC Med Res Methodol. 2012;12:152. doi:10.1186/14712288-12-152.

34. Dequen P, Lorigan P, Jansen JP, van Baardewijk M, Ouwens MJ, Kotapati S. Systematic review and network meta-analysis of overall survival comparing $3 \mathrm{mg} / \mathrm{kg}$ ipilimumab with alternative therapies in the management of pretreated patients with unresectable stage III or IV melanoma. Oncologist. 2012;17:1376-85. doi:10.1634/ theoncologist.2011-0427.

35. Ouwens MJ, Philips Z, Jansen JP. Network meta-analysis of parametric survival curves. Res Synth Methods. 2010;1:258-71. doi:10.1002/jrsm. 25.

36. Hersh EM, O'Day SJ, Powderly J, Khan KD, Pavlick AC, Cranmer LD, et al. A phase II multicenter study of ipilimumab with or without dacarbazine in chemotherapy-naive patients with advanced melanoma. Invest New Drugs. 2011;29:489-98. doi:10.1007/s10637-009-9376-8.
37. Hamid O, Schmidt H, Nissan A, Ridolfi L, Aamdal S, Hansson J, et al. A prospective phase II trial exploring the association between tumor microenvironment biomarkers and clinical activity of ipilimumab in advanced melanoma. J Transl Med. 2011;9:204. doi:10.1186/1479-58769-204.

38. Squibb B-M. An exploratory study to determine potential predictive markers of response and/or toxicity in patients with unresectable stage III or IV malignant melanoma randomized and treated with ipilimumab (MDX-010/BMS-734016) at two dose levels. http://ctr.bms.com/pdf/CA184-004ST.pdf. Accessed 16 August 2016.

39. Wolchok JD, Neyns B, Linette G, Negrier S, Lutzky J, Thomas L, et al. Ipilimumab monotherapy in patients with pretreated advanced melanoma: a randomised, double-blind, multicentre, phase 2, dose-ranging study. Lancet Oncol. 2010;11:155-64. doi:10.1016/S1470-2045(09)70334-1.

40. Squibb B-M. A randomized, double-blind, multi-center, phase II fixed dose study of multiple doses of ipilimumab (MDX-010) monotherapy in patients with previously treated unresectable stage III or IV Melanoma. Final Clinical Study Report for Study CA184022. http://ctr.bms.com/pdf/CA184022\%20ST.pdf. Accessed 16 August 2016.

41. Long GV, Menzies AM, Nagrial AM, Haydu LE, Hamilton AL, Mann GJ, et al. Prognostic and clinicopathologic associations of oncogenic BRAF in metastatic melanoma. J Clin Oncol. 2011;29:1239-46. doi:10.1200/JCO.2010.32.4327.

42. Thomas NE, Edmiston SN, Alexander A, Groben PA, Parrish E, Kricker A, et al. Association between NRAS and BRAF mutational status and melanoma-specific survival among patients with higher-risk primary melanoma. JAMA Oncol. 2015;1:359-68. doi:10.1001/jamaoncol.2015.0493.

43. Menzies AM, Long GV. Recent advances in melanoma systemic therapy. BRAF inhibitors, CTLA4 antibodies and beyond. Eur J Cancer. 2013;49:3229-41. doi:10.1016/j.ejca.2013.06.027.

44. Hugdahl E, Kalvenes MB, Puntervoll HE, Ladstein RG, Akslen LA. BRAF-V600E expression in primary nodular melanoma is associated with aggressive tumour features and reduced survival. $\mathrm{Br} \mathrm{J}$ Cancer. 2016;114:801-8. doi:10.1038/bjc.2016.44.

45. Ugurel S, Thirumaran RK, Bloethner S, Gast A, Sucker A, Mueller-Berghaus J, et al. B-RAF and N-RAS mutations are preserved during short time in vitro propagation and differentially impact prognosis. PLoS One. 2007;2:e236. doi:10.1371/ journal.pone.0000236. 
46. Moreau S, Saiag P, Aegerter P, Bosset D, Longvert C, Helias-Rodzewicz $Z$, et al. Prognostic value of BRAF(V(6)(0)(0)) mutations in melanoma patients after resection of metastatic lymph nodes. Ann Surg Oncol. 2012;19:4314-21. doi:10.1245/s10434-0122457-5.
47. Nowara E, Nieckula J, Badora-Rybicka A, Rajczykowski M, Huszno J. Impact of BRAF V600 mutation in metastatic cutaneous melanoma patients treated with systemic therapy on brain metastases and survival. Pigment Cell Melanoma Res. 2015;28:803. doi:10.1111/pcmr.12419. 